\title{
Favourable outcome of thrombotic thrombocytopenic purpura despite delayed
}

\author{
presentation
}

Osama Abdulla ${ }^{1}$, Manal Mohammed ${ }^{1}$, and Hyder Osman Mirghani²

1. King Fahd Specialist Hospital, Tabuk Saudi Arabia

2. Faculty of Medicine, University of Tabuk, Tabuk, Saudi Arabia

\section{CASE STUDY}

Please cite this paper as: Abdulla OS, Manal SM, Mirghani HO. Favourable outcome of thrombotic thrombocytopenic purpura despite delayed presentation. AMJ 2019;12(11):296-298.

https://doi.org/10.35841/1836-1935.12.11.296-298

Corresponding Author:

Hyder O Mirghani

Faculty of Medicine, University of Tabuk, Tabuk, Saudi Arabia

Email: s.hyder63@hotmail.com

\section{ABSTRACT}

Delayed presentation of thrombotic thrombocytopenic purpura (TTP). We report a female patient presenting with a headache and dizziness for two weeks, her past medical history is notable for epistaxis otherwise unremarkable. On examination she was pale with a tinge of jaundice. She was vitally stable, other systems were unremarkable. A peripheral blood smear showed evidence of microangiopathic haemolytic anaemia (MAHA) and thrombocytopenia. No evidence of organ dysfunction. Plasma exchange and corticosteroids were started with dramatic response. This case illustrates the efficacy of plasmapheresis in late TTP.

\section{Key Words}

Thrombotic thrombocytopenic purpura, plasmapharesis

\section{Implications for Practice:}

\section{What is known about this subject?}

TTP is a rare disorder characterized by a histopathologic finding of microvascular thromboses and end organ damages. Timely institution of plasmapharesis is vital for treatment.

2. What new information is offered in this case study? Plasmapharesis and steroid were effective even in delayed severe presentation.

3. What are the implications for research, policy, or practice?

Timely institution of plasmapharesis and steroid are critical for prognosis of TTP.

\section{Background}

Thrombotic microangiopathies (TMAs) are characterized by a histopathologic finding of microvascularthromboses and end organ damages. The typical clinical presentation is microangiopathic haemolytic anaemia accompanied by thrombocytopenia with varying degrees of organ ischemia. ${ }^{1}$

Historically, TTP was diagnosed by the clinical pentad of fever, thrombocytopenia, microangiopathic haemolytic anaemia, neurological symptoms, and renal insufficiency that are only present in less than 10 per cent of patients with an acute TTP. ${ }^{2}$

Intracranial haemorrhage, arterial thrombosis, ischemic stroke, myocardial infarction, renal failure, platelets transfusion, and age were perfect discrimination and was predictive of mortality. $^{3}$

Anti-ADAMTS13 IgG antibody and ADAMTS13 antigen levels correlate with outcome in TTP with increased cardiac and neurological involvement and increased mortality. ${ }^{4}$

This case substantiates the favourable outcome of TTP in the absence of organ dysfunction. However, the decision of timely institution of plasma exchange should be overemphasized, and the treating physician must not be deceived by the apparent patient stability. 


\section{Case details}

A 35-year-old Ghanaian woman presented with an intractable headache and dizziness for approximately 14 days before admission. Her past medical history was remarkable for an episode of epistaxis. A headache was throbbing, intermittent, moderately severe and frontal in location. The dizziness started while she was sitting and was aggravated by postural changes.

On physical examination, the patient was stable and not in acute distress. Vital signs were normal. Examinations of eyes were notable for pallor and tinge of jaundice. Neurological examination was normal. There was no evidence of active oral, vaginal or rectal bleeding. Examination of the skin and joints: neither ecchymosis nor purpura. No malar rash or another type of rash was present on sun-exposed areas. No tenderness or deformities were present in the joints.

TTP was suspected as evident by microangiopathic haemolytic anaemia (Figure 1), severe thrombocytopenia, high LDH level, and negative Coombs test.

\section{Discussion}

Without treatment, thrombotic thrombocytopenic purpura is fatal in more than 90 per cent of cases. ${ }^{4}$ Since the introduction of therapeutic plasma exchange in 1980 there was a dramatic reduction in mortality (from 90 per cent to 10-20 per cent) among patients with TTP, however, United States analysis of mortality reported an increasing rate overtime despite the reduction observed after the introduction of plasmapheresis. ${ }^{5}$ Despite proven efficacy and increased availability of therapeutic plasma exchange (TPE), mortality for patients with thrombotic thrombocytopenic purpura (TTP) remains high with a limited understanding about this issue. ${ }^{6}$ Nationwide Analysis from 2005-2014 published by Liu and colleagues found a Decreased length of hospitalization and cost but not mortality among patients who received early plasmapheresis. ${ }^{7}$ Patients with the immune form of the disease that is not secondary to a medical condition (i.e., not the acquired form) and those with the hereditary form respond well to plasma exchange therapy. ${ }^{8}$ Therapeutic plasma exchanged should be instituted even there is uncertainty about the diagnosis because the benefit in the term in mortality reduction overweighs the risk of TPE. ${ }^{9}$ There are several factors predict the outcome from TTP, organ dysfunctions are among the most important, however they are not observed in the current report. Cardiac involvement is a major cause of mortality in patients with thrombotic thrombocytopenic purpura (TTP) in particular for patients with Cardiac troponin-I (CTnl) level of $>0.25 \mu \mathrm{g}$ L(-1) at presentation (The later appears to be an independent factor associated with a three-fold increase in the risk of death or refractoriness). ${ }^{10}$ In contrast to Federici et al. ${ }^{11}$ who concluded that a longer therapeutic delay of plasma exchange (PE) was predictive of resistance to $P E$, Our case demonstrates a favourable outcome despite delayed institution of PE. The absence of Organ dysfunction in patients with TTP predicts a favourable outcome (Figure 2).

Similar dramatic improvement of a patient with late presentation and neurological findings was observed by Ogilvie and colleagues from the USA. The persistent elevation of $\mathrm{LDH}$ and the severe headache observed are pointers of severity in the present review. The addition of steroid to plasmapharesis our case may improve recovery. ${ }^{12-}$ 14

\section{Conclusion}

This case illustrates the efficiency of plasmapheresis and steroid therapy even in late presentation of TTP.

\section{References}

1. George JN, Nester CM. Syndromes of thrombotic microangiopathy. N Engl J Med. 2014;371(7):654-666.

2. Scully $M$, Yarranton $H$, Liesner $R$, et al. Regional UK TTP registry: correlation with laboratory ADAMTS 13 analysis and clinical features. Br J Haematol. 2008;142(5):819826.

3. Alwan F, Vendramin C, VanhoorelbekeK, et al. Presenting ADAMTS 13 antibody and antigen levels predict prognosis in immune-mediated thrombotic thrombocytopenic purpura. Blood. 2017;130(4):466-471.

4. Chaturvedi S, Carcioppolo D, Zhang L, et al. Management and outcomes for patients with TTP: analysis of 100 cases at a single institution. Am J Hematol. 88(7):560-5, 2013

5. Torok TJ, Holman RC, Chorba TL. Increasing mortality from thrombotic thrombocytopenic purpura in the United States--analysis of national mortality data, 19681991. Am J Hematol. 1995;50:84-90.

6. Liu Y, Han H, Wei X, et al. Outcomes of Early Plasmapheresis Vs Late Plasmapheresis in Patients with Thrombocytopenic Thrombotic Purpura: A Nationwide Analysis from 2005-2014. Blood. 2017;130(1):3640. https://doi.org/10.1182/blood.V130.Suppl_1.3640.364 0

7. Goel R, King KE, Takemoto CM, et al. Prognostic riskstratified score for predicting mortality in hospitalized patients with thrombotic thrombocytopenic purpura: 
nationally representative data from 2007 to 2012 . Transfusion. 2016;56(6):1451-8. doi: 10.1111/trf.13586. Epub 2016 Apr 15.

8. Crawley JT, Scully MA. Thrombotic thrombocytopenic purpura: basic pathophysiology and therapeutic strategies. Hematology Am Soc Hematol Educ Program. 2013:292-9, 2013

9. Scully M, Hunt BJ, Benjamin S, et al. British Committee for Standards in $\mathrm{H}$. Guidelines on the diagnosis and management of thrombotic thrombocytopenic purpura and other thrombotic microangiopathies. $\mathrm{Br} J$ Haematol. 2012;158:323-35.

10. Benhamou Y, Boelle PY, Baudin B. Cardiac troponin-I on diagnosis predicts early death and refractoriness in acquired thrombotic thrombocytopenic purpura. Experience of the French Thrombotic Microangiopathies Reference Center. J Thromb Haemost. 2015;13(2):293302.

11. Federici L, Klouche K, Amigues L, et al. Outcome and prognosis of severe thrombotic microangiopathies treated by plasma exchange. Med Sci Monit. 2006;12(7): CR302-7.

12. Ogilvie J, Singh J. A dramatic recovery in a patient initially expected to die of TTP \& its complications. J Community Hosp Intern Med Perspect. 2018 Jun 12;8(3):142-144. doi: 10.1080/20009666.2018.1475186. eCollection 2018.

13. Chaturvedi S, Carcioppolo D, Zhang L, et al. Management and outcomes for patients with TTP: analysis of 100 cases at a single institution. Am J Hematol. 2013;88(7):560-5. doi: 10.1002/ajh.23455. Epub 2013 May 30.

14. Korkmaz S, Keklik M, Sivgin S, et al. Therapeutic plasma exchange in patients with thrombotic thrombocytopenic purpura: a retrospective multicenter study. Transfus Apher Sci. 2013;48(3):353-8. doi: 10.1016/j.transci.2013.04.016. Epub 2013 Apr 18.

\section{PEER REVIEW}

Not commissioned. Externally peer reviewed.

\section{CONFLICTS OF INTEREST}

The authors declare that they have no competing interests.

\section{FUNDING}

The manuscript is self-funded

\section{PATIENT CONSENT}

The authors, Abdulla OS, Manal SM, Mirghani HO, declare that:
1. They have obtained written, informed consent for the publication of the details relating to the patient(s) in this report.

2. All possible steps have been taken to safeguard the identity of the patient(s).

3. This submission is compliant with the requirements of local research ethics committees.

Figure 1: Peripheral blood smear showed schistocytes

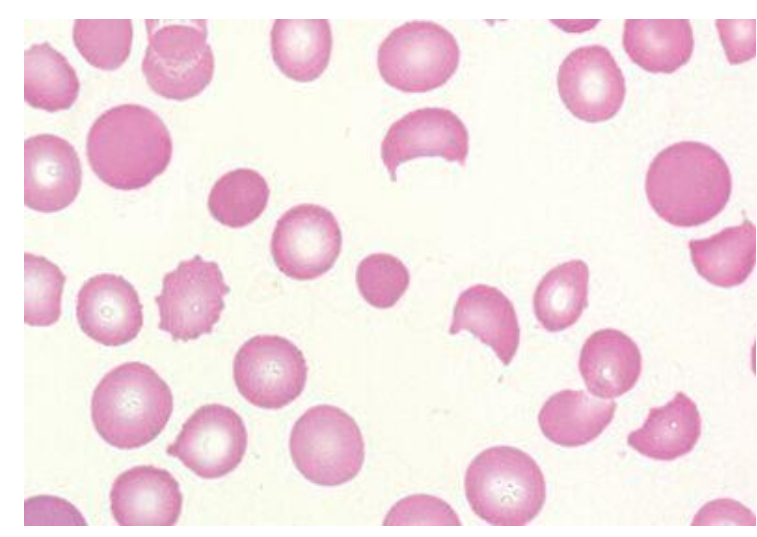

Figure 2: Serial platelet measurement following plasmapharesis

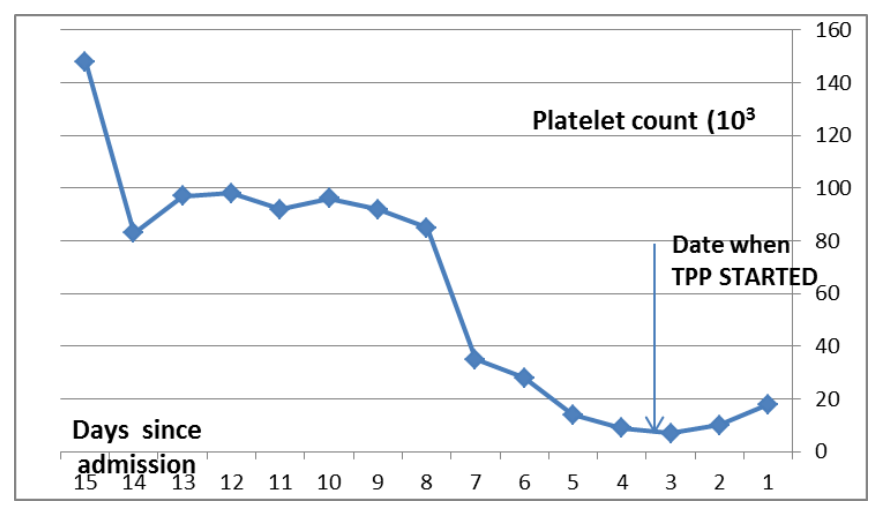

